\title{
高中物理习题教学中学生科学思维的培养策略——以“楞次定律” 习题课教学为例
}

\author{
崔娟 张岩文 刘永军 葛桂贤
}

石河子大学理学院

DOI:10.32629/er.v3i4.2601

[摘 要] 习题教学是培养学生科学思维的一个重要途径。我们以高中物理学中的楞次定律为例, 统计分析了学生出错比较严重的习题,找出了 学生在分析、推理过程中存在的思维障碍,并给出相应的教学建议,希望能为培养学生的科学思维提供帮助。

[关键词] 科学思维; 楞次定律; 习题教学

\section{前言}

“科学思维” 作为物理核心素养的主要内容, 包括对物理学对象内在 规律的认识、模型的构建、科学的推理和分析论证、质疑创新 ${ }^{[1]}$ 。随着课 程改革的推进, 学生科学思维的培养越来越受到了普遍的重视。

物理习题教学是物理教学的重要组成部分, 在培养学生的分析和解决 问题能力、锻炼学生的思维方面起着重要的作用。然而在目前的习题教学 中仍然存在着只重视知识不重视能力的现象, 这种教学模式不能让学生主 动运用物理知识解决实际问题, 也不利于培养学生的科学思维能力 ${ }^{[2]}$ 。楞 次定律作为高中物理阶段最为抽象的物理规律, 因其推理过程复杂, 结论 难理解的特点, 长期以来是教学的难点, 学生在做相关的习题过程中存在 大量的出错现象。如何加深学生对楞次定律的深入理解, 降低学生习题出 错的概率, 同时将科学思维的培养途径运用于楞次定律的习题教学是值得 研究的一个问题。从查阅的文献来看, 目前关于楞次定律的研究大多数是 从内涵和探究实验的角度进行探讨, 而关于楞次定律习题教学研究探讨较 少 ${ }^{[3]}$ 。因此本文通过分析学生容易出错的典型习题, 探讨能够培养学生科 学思维的楞次定律习题课的教学策略, 从而解决学生的思维障碍提高学生 解题效率。

\section{1 学生解题现状及分析}

1. 1 学生答题情况的简单统计

表1 周测试试卷中学生的答题情况

\begin{tabular}{|c|c|c|c|c|c|c|c|c|}
\hline 题号 & 答案 & 满分 & 平均分 & 难度 & 正确率 & 区分度 & 标准差 & 优秀率 \\
\hline $\mathrm{k} 1-1$ & $\mathrm{~A}$ & 4 & 1.676 & 0.419 & $41.911 \%$ & 0.32 & 1.97 & 41.91 \\
\hline $\mathrm{k} 1-2$ & $\mathrm{~A}$ & 4 & 3.069 & 0.767 & $76.733 \%$ & 0.54 & 1.69 & 76.73 \\
\hline $\mathrm{k} 1-3$ & $\mathrm{~B}$ & 4 & 3.519 & 0.88 & $87.982 \%$ & 0.38 & 1.3 & 87.98 \\
\hline $\mathrm{k} 1-4$ & $\mathrm{~A}$ & 4 & 2.126 & 0.532 & $53.159 \%$ & 0.37 & 2 & 53.16 \\
\hline $\mathrm{k} 1-5$ & $\mathrm{~A}$ & 4 & 3.797 & 0.949 & $94.915 \%$ & 0.3 & 0.88 & 94.92 \\
\hline $\mathrm{k} 1-6$ & $\mathrm{D}$ & 4 & 3.328 & 0.832 & $83.205 \%$ & 0.22 & 1.5 & 83.2 \\
\hline $\mathrm{k} 1-7$ & $\mathrm{D}$ & 4 & 1.072 & 0.268 & $26.81 \%$ & 0.37 & 1.77 & 26.81 \\
\hline $\mathrm{k} 1-8$ & $\mathrm{~A}$ & 4 & 2.706 & 0.676 & $67.643 \%$ & 0.5 & 1.87 & 67.64 \\
\hline $\mathrm{k} 1-9$ & $\mathrm{D}$ & 4 & 2.613 & 0.653 & $65.331 \%$ & 0.55 & 1.9 & 65.33 \\
\hline $\mathrm{k} 1-10$ & $\mathrm{C}$ & 4 & 2.706 & 0.676 & $67.643 \%$ & 0.41 & 1.87 & 67.64 \\
\hline $\mathrm{k} 1-11$ & $\mathrm{CD}$ & 6 & 4.128 & 0.688 & $63.02 \%$ & 0.67 & 2.59 & 63.02 \\
\hline $\mathrm{k} 1-12$ & $\mathrm{AB}$ & 6 & 1.322 & 0.22 & $7.704 \%$ & 0.28 & 1.9 & 7.7 \\
\hline $\mathrm{k} 1-13$ & $\mathrm{ABD}$ & 6 & 2.773 & 0.462 & $25.578 \%$ & 0.48 & 2.29 & 25.58 \\
\hline
\end{tabular}

表1给出了石河子地区某中学高二年级11个班级649名学生周测卷中 楞次定律相关选择题的答题情况。从表中可以看到题目的正确率与题目的 难度基本上是对应的。学生正确率较低的题目从低到高依次是第 12 题、13 题、7题, 它们的正确率分别是7. 704\%, 22. 958\%, 25. 578\%, 26. 81\%, 其中单选 题中第7题的错误率最高, 多选题中第 $12 、 13$ 题错误率比较高。

\section{2 学生解题思维障碍分析}

对周测卷中的学生的答题情况进行分析可以发现目前高中物理习题 教学中存在一些问题, 例如, 第 1 题作为对物理学史等概念性知识的考察, 其正确率很低 (41.911\%), 学生主要对测量元电荷的数值的实验、静电常数 $\mathrm{k}$ 的提出、库仑的扭秤实验、卡文迪许的扭秤实验等陈述性知识的了解不 够清楚导致混淆。说明教师在教学中注重讲授大量的习题但忽视了对于基 础的陈述性知识的讲解。第 7 题的正确率也较低 (26.81\%), 学生最容易选错 的是B选项, 通过左手定则学生可以判断线圈所受的力与线圈速度方向垂 直, 所以学生能够对物体一开始作曲线运动做出判断 (由左手定则可以判 断金属环所受到的力与物体的初速度方向不一致), 但随着金属环由于受 到安培力的作用, 其运动速度发生改变, 使得金属环的运动方向逐渐偏向 平行于通电直导线的方向, 此时线圈内的磁通量变化为 0 , 所以此时金属环 中不再有感应电流产生, 也不再受安培力的作用, 金属环沿通电直导线做 匀速直线运动。说明学生对部分考察内容较为熟悉, 但在做题过程中不能 抓住题目的关键信息, 在解题过程中目标和步骤并不明确, 缺乏对物理学 知识本质的理解所以不能统筹所学内容进行解题。也说明了教师在习题教 学中只关注难度较大的题目, 缺乏对基础知识和解题过程的讲解, 使学生 难以应对较灵活的题目。第 12 题中学生的正确率为 $7.704 \%$, 主要出问题的 是 $C$ 选项, 学生以一般的思维来思考 $B$ 点的磁感应强度确实要比 $A$ 点的磁感 应强度小, 那么在该点通电导线所受的安培力 $(F=B I L)$ 也较小, 忽略了在 一般情况下要考虑磁感应强度 $B$ 与导线的夹角, 也就是 $F=B I L \sin \theta$, 所以导 线在 $A 、 B$ 两点所受安培力的大小不能确定。说明学生对习题考察的内容缺 乏自己的思考, 不能在教师讲解完例题后独立完成变式训练。也反应了教 师在习题课中强调解题技巧但忽视了变式训练对学生科学思维培养的重 要作用。

综上, 教师在高中物理习题教学中主要出现的问题有:

(1) 注重讲授大量的习题但忽视了对于基础的陈述性知识的讲解。例 如, 为了加快教学进度教师在习题课中常会在一开始就快速地讲解练习册 
中的习题, 而对新授课中的概念、规律、物理学史等陈述性知识没有进行 简单总结以加深学生印象, 学生对之前学习的新内容不熟悉, 可能会导致 由于基础知识不明确出现错误。

(2) 关注难度较大的题目, 缺乏对基础知识和解题过程的讲解。具体表 现为教师会选择他们认为难度较大的题目进行讲解, 强调解题的关键内容, 但是, 忽视了对基础内容和具体思考过程的讲述, 学生很难从这些题目中 总结出思考此类题目的解题步骤。

(3) 强调解题技巧, 但忽视了变式训练对学生科学思维培养的重要作 用。部分教师注重总结某一类题目的解题技巧, 这些技巧在一定程度上确 实可以加快解题速度, 但这种方式使学生忽视了技巧背后的物理学本质, 而且很多解题技巧都有适用范围, 若只是简单的套用可能会掉入出题人的 陷阱, 所以在习题教学中应当注意以变式训练的方式培养学生的科学思维, 使学生真正理解解题过程并总结出解题技巧。

通过对楞次定律相关习题的分析发现学生在楞次定律这一节内容中
面临的思维障碍首先是对楞次定律的概念认识不清, 其次是对“感应磁场” 和 “原磁场” 的变化分析不够明确, 最后能够通过楞次定律对题目进行初 步分析但不能结合前面所学知识统筹解决问题, 这些思维障碍的出现本质 原因是由于学生和教师对于科学思维的培养不够重视导致学生不能运用 楞次定律分析最后解决问题。

\section{2 习题教学中科学思维的培养}

科学思维能力的培养有助于学生建构个人的思维体系, 形成一套解决 问题的方法和策略, 在面对复杂的科学问题时能有效地提取关键信息, 对 不同的观点能提出质疑和创造性的见解。目前基于学科核心素养导向的高 考物理命题, 也突出了对科学思维能力的考察, 这就要求教师在平时的习 题教学中要注重落实。习题教学是巩固知识、培养能力形成科学思维的一 种重要的课堂教学组织形式, 习题课的教学可以从内在规律的认识、模型 的构建、分析与综合和质疑与创新四个方面来培养学生科学思维, 如图 1 所示。

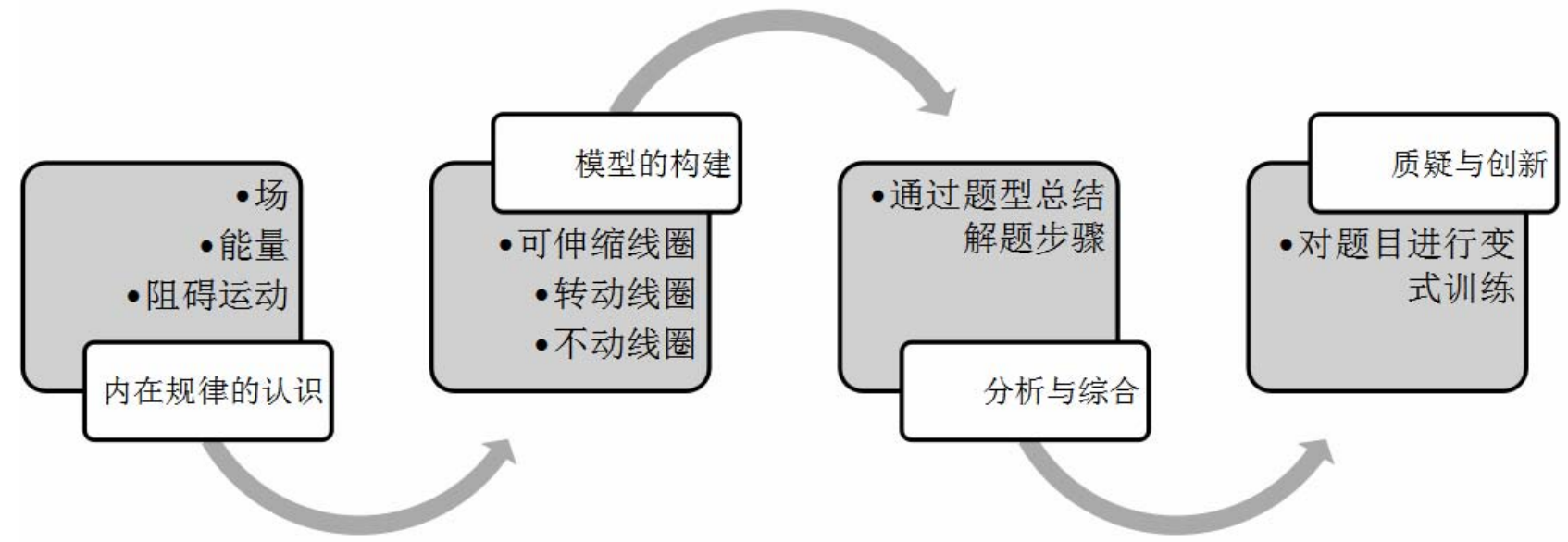

图1 科学思维培养的四个方面

2.1在习题教学中引导学生以物理学视角认识客观事物的内在规律

在习题教学过程中教师首先应当从物理学的视角以教师复述或提 问的方式总结新授课中已学习的概念或规律的内在规律, 使学生对基础 知识有更加全面深入的理解。而不是刻意地去总结一些所谓的解题技巧, 过分强调技巧可能会弱化学生对客观事物本质属性的思考。结合相关知 识点中易错内容的讲解可以使学生发现自身在新授课中对知识点内涵 的错误或不完整的理解, 形成对物理知识内在规律的深刻认识, 在一般 的习题教学中也应当强化对相关基础概念的强化, 这是培养学生科学思 维的第一步。

以楞次定律为例, 对楞次定律进行 “引起感应电流磁通量变化” 和 “阻 碍” 两个方面来理解, “引起感应电流磁通量” 意味着 “原磁场” 的变化 引起对应线圈内的磁通量的变化, 在部分题目中可能会偷换概念, 提醒学 生注意; “变化” 意味着 “原磁场” 变化才能够产生感应电流, 否则就不产 生感应电流, 也就不会受到安培力的作用; “阻碍” 要明白 “谁阻碍谁” (感 应电流的磁场阻碍原磁通量的变化) ; “阻碍什么” (阻碍的是原磁通量的 变化而不是磁通量本身); “怎样阻碍” (当穿过闭合回路的磁通量增加时, 感应电流的磁场方向总是与原磁场方向相反; 当穿过闭合回路的磁通量减 小时, 感应电流的磁场方向总是与原磁场方向相同 $)^{[4]}$ 。通过两个方面的讲 解使学生对楞次定律的内在规律有了更深刻的理解, 这从内在规律的理解 方面培养了学生的科学思维。

2. 2 在习题教学中提高学生的物理建模思维意识
对物理学实际过程进行简化、经过抽象后建立的可以用数学进行描述 的对象我们称为物理模型。在高中物理中为了满足习题教学的需要我们对 物理模型进行了外延, 建立了很多不是非常严格的物理模型, 这些物理模 型是培养学生科学思维的重要工具。在习题讲解完成后应当引导学生结合 类似的例子进行物理模型的建构, 物理模型的建构过程也是学生对这一类 题型的内化过程, 并且学生可以克服在习题中容易形成的思维障碍。在解 决物理问题时能够对物理情景进行简化并建立正确的物理模型是解决物 理问题的重要方法, 习题教学能够为培养学生的建模思想提供平台, 所以 在习题教学中教师要设计能够让学生自己参与建模的教学环节, 使学生发 现题目中的异同点, 总结相关物理习题的模型, 为下一次解决此类问题做 好准备。通过建模的过程可以培养学生根据事实证据进行科学推理的科学 思维。

以楞次定律的习题教学为例, 在例题的讲解过程中, 教师可以引导 学生建立楞次定律习题的模型, 结合第 7 题和磁通量变化是由磁体或线 圈的运动产生的例题分析, 线圈与磁铁之间的相互作用表现出的 “来拒 去留” 或 “增斥减吸” 的特点。结合第 12 题的变式和相关线圈大小可变 化的习题, 分析关于楞次定律的二级结论 “增反减同” 和 “增缩减扩” 的应用条件。结合第 13 题和铜制金属环从条形磁铁的正上方由静止开始 下落的习题, 金属环由静止开始下落的过程中, 减少的重力势能一部分 转化为动能, 一部分转化为内能, 机械能减小。从能量的角度分析, 楞次 定律的本质就是在 “阻碍” 的过程中, 发生能量的转化。综上, 关于楞次 
定律习题的模型建构主要分为三类, 可伸缩线圈、磁场中转动的线圈、 不动线圈

2.3 在习题教学中注重培养学生分析与综合的思维能力

分析和综合各类模型包含的更深层次的内涵, 并总结关于楞次定 律的解题步骤, 是培养学生科学思维的核心内容, 分析与综合阶段的完 成可以在讲解完三种模型的习题后进行。教师通过讲述相关经典习题 的思考方向和解题过程, 使学生对这类习题的解决过程有了相对清晰 的认识, 此时教师应当引导学生分析和综合各类模型的内涵, 并总结相 关习题的解题步骤, 学生在遇到这类习题时能够对题目有综合的认识, 解决问题的目标明确思路清晰, 避免出现之前的思维障碍而掉入出题 人的陷阱。

不论从场的角度还是从能量的角度, 或是从运动的角度来看, 对于楞 次定律的理解都是从 “阻碍” 出发, 将物理情景对应于物理模型, 寻找适合 的解题技巧, 最终又回到 “阻碍” 得到最后的结论, 这一结论的得出可以有 多种教学形式, 比如学生讨论并以说课的形式回答后由教师补充、教师展 示题目的变式让学生对比产生认知冲突, 这是结合所学知识并运用楞次定 律解决其他问题的前提, 也是训练学生分析综合能力、提高学生科学思维 的重要途径。其次, 用解决此类题型的步骤:

(1) 确定原磁场的方向;

(2) 确定磁通量的变化;

(3) 判断感应磁场的方向;

(4) 确定感应电流的方向;

(5) 结合之前所学内容解决问题。

分析题目属于哪一类模型。最后, 运用楞次定律分析磁通量变化情 况, 感应电流产生情况和研究对象受力情况。

2. 4在习题教学中鼓励学生深入挖掘大胆质疑与创新

在习题教学中结合相关例题的变式练习和相关知识点在日常生活中 应用的讲述使学生在理解教学内容的基础上进行质疑和创新, 这一环节的 设计需要教师对学生给予肯定和鼓励, 使学生敢于表达个人观点, 并从更 高的视角对物理知识进行质疑和创新, 实现了对物理知识的应用, 逐渐形 成对物理学本质的认识, 提高了解决实际问题的能力, 最终达到培养学生 科学思维的目的。
在楞次定律的习题教学尾声阶段, 要引导学生对建立的图示提出质疑 或创新, 只有学生在完全理解内容的情况下才能从更高的视角对图示提出 针对性和创造性的见解。质疑与创新的实际操作以变式训练和解释生活现 象为主。以第 7 题为例, 如果直导线电流 I 有限, 金属环初始速度较大, 则金 属环最后一定会顺着直导线方向做匀速直线运动? 以 12 题为例, 若改变题 目给定条件, 再在环形线圈外加一个反向电流的线圈后分别在 $A, B, C$ 三点 的通电直导线所受的安培力会发生变化, 外围大线圈形成的磁场会抵消部 分小线圈内的磁场, 所以 $A$ 点的安培力会减小, 但 $B 、 C$ 两点处于小线圈和大 线圈之间磁场叠加, 导线在该点所受安培力增加。另外, 金属探测器内含有 通电线圈, 当它靠近金属材料的物体时为何会发出警报? 电磁炉为何能够 加热食物? 电磁冲击钻的钻头突然运动的原因是什么? 这些问题都可以 鼓励学生利用楞次定律来解释。

\section{3 结语}

以楞次定律为例, 在习题教学中从内在规律的认识、模型的构建、分 析与综合以及质疑与创新四个方面入手不仅能够使学生对知识的理解更 加深入, 同时更重要的是能够培养学生的科学思维, 提高学生发现问题、提 出问题和解决问题的能力, 引导学生从物理学的视角认识事物的本质属性, 通过经验事实建构物理模型, 基于事实依据和科学推理提出质疑和创新性 见解, 在解决问题的过程中培养学生的科学思维。

\section{[参考文献]}

[1]周孝明.对楞次定律 “来拒去留” “增缩减扩” 的一些思考 [J].湖南 中学物理,2018,33(02):92-93.

[2]刘湘衡.基于学生科学思维培养的高中物理高效课堂建构[J].新课 程研究(中旬刊),2019,(02):18-19.

[3]黄伟.提升学生科学思维能力的物理教学探讨一——“楞次定律” 教学为例[J].物理之友,2019,35(06):21-22.

[4]万大林, 黄绍书. 楞次定律的多角度阐释及其应用 [ J]. 物理通 报,2019,(04):29-31.

\section{作者简介:}

崔娟(1995--), 女, 汉族, 甘肃省白银人, 石河子大学理学院学科教 学(物理)硕士研究生, 研究方向: 学科教学(物理)。 